\title{
Multi-resolution joint auto correlograms for content-based image retrieval
}

\begin{abstract}
The past few years have seen a major development in Content-based Image Retrieval (CBIR) due to the needs by various fields in accessing visual data, particularly images. As a result, several techniques have been developed to allow image databases to be queried by their image content. Colour Auto Correlogram (CAC) is one of the promising colour descriptors used to extract and index image features effectively. However, the conventional CAC and most of its advancements are only based on single image feature, not sensitive to scale, and computed in the spatial domain. A new method for CBIR has been introduced by allowing multiple local image features to represent an image rather than just colour and extracting them at different level of the image sub-bands to provide different physical structures of the image in the frequency domain. The Ridgelet transform is performed on the RGB colour space and the greyscale version of the image to provide the multi-resolution levels. Colour feature is extracted from the Ridgelet coefficients of the RGB colour space while other image features such as gradient magnitude, rank, and texturedness are extracted from the Ridgelet coefficients of the grey-scale image. Each of these image features is quantised and the auto correlogram is then performed on the respective quantised image feature coefficients. Retrieval experiments conducted on 1000 SIMPLIcity image database has demonstrated that the proposed Multiresolution Joint Auto Correlograms (MJAC) has achieved a significant improvement in effectiveness compared to the traditional $\mathrm{CAC}$ and several of its advancements.
\end{abstract}

Keyword: CAC; CBIR; Colour descriptor; MJAC; Ridgelet transform 